\title{
The relationship between poor nutritional status and progression of aortic calcification in patients on maintenance hemodialysis
}

Teppei Okamoto', Shingo Hatakeyama ${ }^{2 *}$ (D) Hirotake Kodama², Hirotaka Horiguchi ${ }^{2}$, Yuka Kubota $^{2}$, Koichi Kido ${ }^{2}$, Masaki Momota ${ }^{2}$, Shogo Hosogoe ${ }^{1,2}$, Yoshimi Tanaka ${ }^{1,2}$, Tooru Takashima ${ }^{1}$, Fumitada Saitoh' ${ }^{1}$, Tadashi Suzuki ${ }^{3}$ and Chikara Ohyama ${ }^{2,4}$

\begin{abstract}
Background: Although aortic calcification has a significant negative impact on prognosis in patients on hemodialysis (HD), risk factors for aortic calcification progression remain unclear. The aim of this study was to investigate the relationship between malnutrition and aortic calcification progression in patients on HD.

Methods: Between April 2015 and October 2016, we treated 232 patients on HD. Of those, we retrospectively evaluated data from 184 patients who had had regular blood tests and computed tomography (CT) scans. The abdominal aortic calcification index (ACI) was quantitatively measured by abdominal $C T$. Nutritional status was evaluated using the Geriatric Nutritional Risk Index (GNRI). A normalized treatment ratio of functional urea clearance was evaluated by Kt $\mathrm{N}$. The difference in $\mathrm{ACl}$ values between 2015 and 2016 was evaluated as a $\Delta \mathrm{ACl}$, and patients were stratified into two groups according to $\triangle \mathrm{ACl}$ value: high ( $\geq 75$ th percentile, $\Delta \mathrm{ACl}$-high group) and low $(<75$ th percentile, $\triangle \mathrm{ACl}$-low group). Variables such as age, sex, comorbidities, dialysis vintage, serum data, and GNRI were compared between $\Delta \mathrm{ACl}$-high and $\triangle \mathrm{ACl}$-low patients. Factors independently associated with a higher $\Delta \mathrm{ACl}$ progression ( $\triangle \mathrm{ACl} \geq 75$ th percentile) were determined using multivariate logistic analysis.
\end{abstract}

Results: Median values of ACls in 2015 and 2016 were 40.8 and 44.6\%, respectively. Of 184 patients, 125 (68\%) patients experienced $\mathrm{ACl}$ progression for 1 year. The median $\triangle \mathrm{ACl}$ and 75 th percentile of $\triangle \mathrm{ACl}$ were $2.5 \%$ and $5.8 \%$, respectively. The number of patients in the $\Delta \mathrm{ACl}$-low and $\triangle \mathrm{ACl}$-high groups were $128(70 \%)$ and $56(30 \%)$, respectively. There were significant differences in sex, presence of diabetic nephropathy, HD vintage, serum albumin, serum phosphate, C-reactive protein, intact parathyroid hormone, Kt/N, and GNRI. Multivariate logistic regression analysis revealed that independent factors associated with a higher $\triangle \mathrm{ACl}$ progression were male sex, serum phosphate levels, HD vintage, and GNRI of $<90$.

Conclusions: Our results suggest that poor nutritional status is an independent risk factor for the progression of aortic calcification. Nutrition management may have the potential to improve progression of aortic calcification in patients on HD.

Trial registration: UMIN Clinical Trials Registry UMIN000028050.

Keywords: Malnutrition, Geriatric nutritional risk index, Aortic calcification progression, Hemodialysis

\footnotetext{
* Correspondence: shingoh@hirosaki-u.ac.jp

${ }^{2}$ Department of Urology, Hirosaki University Graduate School of Medicine, 5

Zaifu-chou, Hirosaki 036-8562, Japan

Full list of author information is available at the end of the article
} 


\section{Background}

Arterial calcification is a typical phenomenon of patients with chronic kidney disease (CKD) and those undergoing hemodialysis (HD) [1, 2]. Arterial calcification is strongly related to all-cause and cardiovascular mortality and morbidity in patients with CKD [3, 4]. Both classical and non-classical risk factors have been implicated in vascular calcification progression among patients undergoing HD. Classical risk factors which can predict coronary heart disease outcomes are male sex, hypertension, smoking, and diabetes mellitus [5]. Non-classical risk factors (uremia-related factors) such as serum phosphate, calcium phosphate product, and intact parathyroid hormone (i$\mathrm{PTH}$ ) were significantly related to arterial calcification in HD patients [6-8]. There are few promising treatments to decrease arterial calcification [9]. Therefore, prevention is crucial to reduce the mortality and morbidity of patients on maintenance HD.

Malnutrition is highly prevalent among patients on maintenance HD. Malnutrition is significantly associated with arterial sclerosis, cardiovascular disease (CVD), and total mortality of HD patients [10]. There exist several tools to evaluate malnutrition. Of these, the Geriatric Nutrition Risk Index (GNRI), calculated using serum albumin, height, and dry weight, is a simple and accurate nutritional indicator for patients on maintenance HD [11]. Several reports have suggested that GNRI is a significant predictor of mortality from CVD [10]. Furthermore, a recent study reported that GNRI was significantly related to the severity of aortic calcification in CKD patients not on HD [12]. However, the relationship between malnutrition and progression of vascular calcification remains unclear. Here we aimed to investigate the relationship between progression of aortic calcification and malnutrition in patients on maintenance HD.

\section{Methods}

\section{Study design}

This was a retrospective, single center, observational study. The study was conducted in accordance with the ethical standards of the Declaration of Helsinki and was approved by the Ethics Committee of Hirosaki University Graduate School of Medicine (authorization number 2016-225). The participants in this study provided their verbal informed consent, and it was recorded in medical chart. Pursuant to the provisions of the ethics committee and ethics guidelines in Japan, written consent was not required in exchange for public disclosure of study information in the case of retrospective and/or observational study using materials such as existing documentation. The ethics committees in Hirosaki University School of Medicine approved this consent procedure. The study information was open for public referral at http:// www.med.hirosaki-u.ac.jp/ uro/html/IRB/IRBdoc.html.

\section{Patient selection}

Between April 2015 and October 2016, we treated 232 patients who had undergone $3-4 \mathrm{~h}$ of maintenance HD or online hemodiafiltration using a dialysate containing $3.0 \mathrm{mEq} / \mathrm{L}$ calcium three times a week at the Oyokyo Kidney Research Institute in Aomori, Japan. Almost all patients had undergone annual abdominal computed tomography (CT) scans to detect incidental renal tumor and other malignancies. Of these, we excluded patients with severe aortic calcification, including calcification extending along the entire length and almost all of the circumference of the abdominal aorta, or who had an inadequate interval ( $\leq 10$ months) of abdominal CT scans. Finally, we selected 184 patients who had undergone adequate abdominal CT scans (Fig. 1).

\section{Evaluation of outcome variables}

Diabetic nephropathy (DMN) was defined as a cause of CKD. Current smoking was defined as smoking at least one cigarette during the study period. Previous history of CVD was defined as having any previous description of ischemic heart disease, cerebrovascular accident, or peripheral arterial disease recorded in the patients' medical records. Every two months, patients underwent a routine laboratory exam before each HD session. We obtained laboratory data for serum phosphate, serum albumin, serum calcium, C-reactive protein (CRP), and iPTH between April 2015 and October 2016. We used the mean blood pressure of six dialysis sessions on the day of blood and serum test as a representative value of blood pressure. Hypertension (HTN) was defined as having a systolic blood pressure of $\geq 140$ and/or a diastolic blood pressure of $\geq 90 \mathrm{mmHg}$. Serum calcium level was

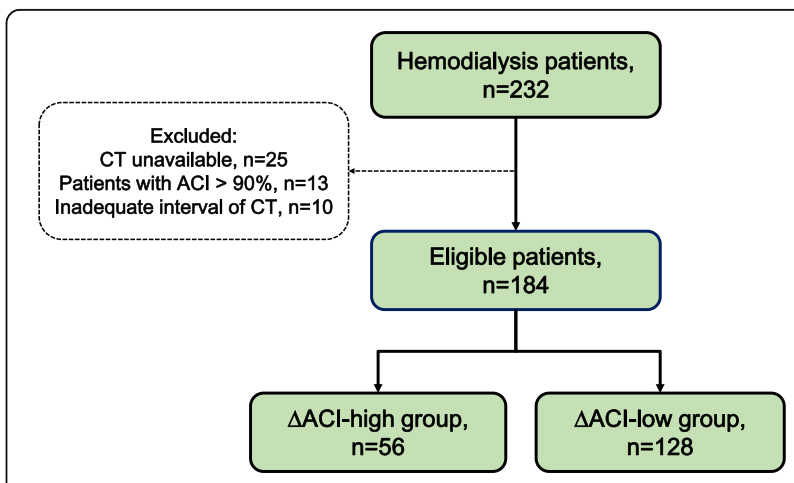

Fig. 1 Patient selection and classification. We treated 232 hemodialysis (HD) patients who underwent 3-4 h-hemodialysis sessions 3 times/week. Of these, we excluded 25 patients with abdominal computed tomography (CT) unavailable. We excluded patients with severe $\mathrm{AC}(\mathrm{ACl}>90 \%)$ in 2015, with an inadequate interval ( $\leq 10$ months) of abdominal $C T$ scans. Finally, the remaining $184 \mathrm{HD}$ patients were included. We divided patients into two groups according to the 75 th percentile of $\triangle \mathrm{ACl}: \triangle \mathrm{ACl}$-high $(\triangle \mathrm{ACl}$ $\geq 5.8 \%)$ or $\Delta \mathrm{ACl}$-low $(\triangle \mathrm{ACl}<5.8 \%)$ 
corrected using the following formula: corrected calcium $=$ total calcium $+0.8 \times(4-$ serum albumin $)$, if serum albumin level was $<4.0 \mathrm{~g} / \mathrm{dL}$. The normalized dialysis dose $(\mathrm{Kt} / \mathrm{V})$ was calculated using the following equation: $\mathrm{Kt} / \mathrm{V}$ $=-\mathrm{Ln}(\mathrm{Ct} / \mathrm{Co}-0.008 \times \mathrm{t})+(4-3.5 \times \mathrm{Ct} / \mathrm{Co}) \times \Delta \mathrm{BW} / \mathrm{BW}$. $\mathrm{Ct}$ divided by Co represents the ratio of post-dialysis to pre-dialysis serum urea nitrogen, $t$ represents dialysis time, and $\mathrm{W}(\mathrm{kg})$ represents post-dialysis body weight.

\section{Abdominal aortic calcification index ( $\mathrm{ACl})$}

We used CT scans (SOMATOM Perspective, Siemens Healthcare, Tokyo, Japan) to evaluate abdominal aortic calcification in all subjects. Images were obtained with a 5-mm slice thickness. Abdominal aortic calcification was semi-quantitatively measured from CT images of the area above the common iliac artery bifurcation by conducting 10 scans at $5-\mathrm{mm}$ intervals, as described elsewhere [13]. Measurement of abdominal aortic calcification in 2015 and 2016 was performed simultaneously. Abdominal aortic calcification index (ACI, \%) represents the calcification proportion in 12 sectors. ACI was calculated using following formula: $\mathrm{ACI}=$ (total score for calcification on all slices) $/ 12 / 10 \times 100(\%)$. We defined ACI values of $>90 \%$ as severe aortic calcification. The difference in ACI values between 2015 and 2016 ( $\triangle \mathrm{ACI}$ ) was calculated by subtracting the ACI value in 2016 (ACI-2016) from that in 2015 (ACI-2015). All procedures were conducted by a single physician before collecting patients' clinical data and background information. We evaluated median ACI-2015, ACI-2016, $\triangle \mathrm{ACI}$, and 75th percentiles of $\triangle \mathrm{ACI}$. Patients were divided into 275 th percentile of $\triangle \mathrm{ACI}$ ( $\triangle \mathrm{ACI}$-high group) and $<75$ th percentile of $\triangle \mathrm{ACI}(\triangle \mathrm{ACI}$-low group).

\section{GNRI}

The GNRI is calculated by the following formula: GNRI $=14.89 \times$ serum albumin $(\mathrm{g} / \mathrm{dL})+41.7 \times$ (body weight $/$ ideal body weight). Ideal body weight was calculated from height and using a body mass index $\left(\mathrm{kg} / \mathrm{m}^{2}\right)$ of 22 . We used mean GNRI between April 2015 and October 2016 as a representative value.

\section{Comparison}

Variables such as age, sex, comorbidities, dialysis vintage, serum data, and GNRI were compared between patients in the $\triangle \mathrm{ACI}$-high and $\triangle \mathrm{ACI}$-low groups. Factors independently associated with a higher $\triangle \mathrm{ACI}$ progression ( $\triangle \mathrm{ACI} \geq 75$ th percentile) were determined using multivariate logistic regression analysis.

\section{Statistical analysis}

Statistical analyses were conducted using SPSS version 22.0 (IBM Corporation, Armonk, NY, USA). Categorical variables (such as sex) are presented as percentages.
Continuous variables with a normal distribution and are expressed as the mean (standard deviation; SD); those with a non-normal distribution are expressed as the median (interquartile range; IQR). Wilcoxon signed-rank test was performed to compare ACI-2015 and ACI-2016 values. Sex $(0=$ female, $1=$ male $)$, HTN $(0=$ absence, $1=$ presence), DMN ( $0=$ other, $1=$ presence), current smoking $(0=$ absence, $1=$ presence $)$, and GNRI $<90(0=a b$ sence, $1=$ presence) were included as binary variables in the model. Comparisons between $\triangle \mathrm{ACI}$-high and $\triangle \mathrm{ACI}$ low groups were performed using Fisher's exact test or Chi-square test, Student's $t$-test (normally distributed data), and Mann-Whitney $U$-test (non-normally distributed data). Multivariate logistic regression analysis was conducted to evaluate an independent predictor for higher $\triangle \mathrm{ACI}$ progression $(\triangle \mathrm{ACI} \geq 75$ th percentile). Based on previous studies, well-known aortic calcification progression factors such as HD vintage, HTN, i-PTH, serum phosphate, corrected calcium level, DMN, sex, age, and current smoking were included in multivariable models. Odds ratios (ORs) with 95\% confidence intervals (CIs) associated with each factor were calculated after adjusting for potentially confounding factors. Probability $(P)$ values $<0.05$ were considered statistically significant.

\section{Results}

\section{Patient classification}

This retrospective study included 184 patients (108 males, 76 females) undergoing HD (Fig. 1). The median age was 66 [interquartile range (IQR), 58-76] years. The mean follow-up period was 11.8 [standard deviation (SD), 0.5] months. Median ACI-2015 and ACI-2016 were $40.8 \%$ (IQR, 15.8-70.2) and 44.6\% (IQR, 20.0$72.5)$, respectively. Of the 184 patients, 125 (68\%) experienced aortic calcification progression for 1 year. The ACI value in 2016 was significantly increased compared to that in $2015(P<0.001)$ (Fig. 2$)$. Median $\triangle \mathrm{ACI}$ and 75th percentiles of $\triangle \mathrm{ACI}$ were $2.5 \%$ and $5.8 \%$, respectively. Numbers of patients in the $\triangle \mathrm{ACI}$-high and $\triangle \mathrm{ACI}$ low groups were $56(30 \%)$ and $128(70 \%)$, respectively.

\section{Comparison of clinical characteristics between patients in $\triangle \mathrm{ACl}$-high and $\triangle \mathrm{ACl}$-low groups}

The clinical characteristics and laboratory data of patients in the $\triangle \mathrm{ACI}$-high and $\triangle \mathrm{ACI}$-low groups are displayed in Table 1 . There were no significant differences in age, prevalence of HTN, or current smoking, or corrected calcium level between the two groups. The proportions of male patients and patients with DMN were significantly higher in the $\triangle \mathrm{ACI}$-high group than in the $\triangle \mathrm{ACI}$-low group (Fig. 3a). Patients in the $\triangle \mathrm{ACI}$-high group had significantly higher levels of serum phosphate $(P=0.005$, Fig. 3b), i-PTH $(P=0.031$, Fig. $3 c)$, and CRP $(P=0.015$, Fig. $3 \mathrm{~d})$ than those in the $\Delta \mathrm{ACI}-$ low group. 


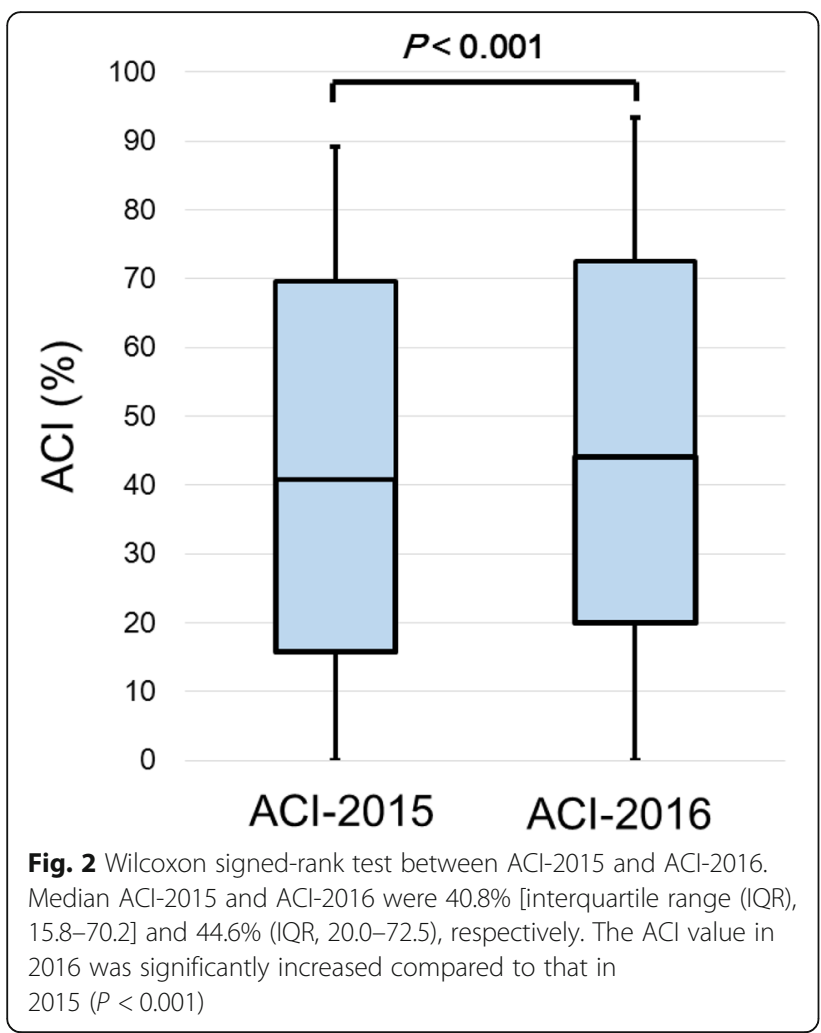

In addition, patients in the $\triangle \mathrm{ACI}$-high group had significant shorter HD vintage $(P<0.001$, Fig. 3e), lower serum albumin level $(P=0.004$, Fig. 3f $)$, lower Kt/V $(P<0.001$, Fig. 3g), and lower GNRI $(P=0.002$, Fig. $3 \mathrm{~h})$ than those in the $\triangle \mathrm{ACI}$-low group. The proportion of patients with poor nutritional status (GNRI <90) was significantly higher in the $\triangle \mathrm{ACI}$-high group than that in the $\triangle \mathrm{ACI}-$ low group (55\% vs. $28 \%, P<0.001)$ (Fig. 4 ).

\section{Independent risk factors for development of high $\triangle \mathrm{ACl}$}

Independent risk factors for an $\mathrm{ACI}$ progression rate greater than the 75th percentile were evaluated by multivariate logistic regression analysis (Table 2). GNRI $<90$ $(\mathrm{OR}=4.17 ; 95 \% \mathrm{CI}=1.79-9.71)$, male sex $(\mathrm{OR}=3.29$; 95\% $\mathrm{CI}=1.27-8.53)$, serum phosphate $(\mathrm{OR}=1.71 ; 95 \%$ $\mathrm{CI}=1.18-2.47)$, and $\mathrm{HD}$ vintage $(\mathrm{OR}=0.99 ; 95 \% \mathrm{CI}=$ 0.98-0.99) were selected as independent risk factors for an ACI progression rate greater than the 75th percentile, after accounting for confounders of aortic calcification progression such as age, DMN, current smoking, HTN, i-PTH, and corrected calcium level (Fig. 5).

\section{Discussion}

In the present study, we investigated clinical parameters that were associated with aortic calcification progression, and found that male sex, serum phosphate level, HD vintage, and GNRI $<90$ were significant factors for aortic calcification progression in patients on maintenance HD.
The key finding was that poor nutritional status was a critical factor for progression of aortic calcification. To the best of our knowledge, this is the first report to demonstrate the relationship between nutritional status and aortic calcification progression in patients on maintenance HD.

Malnutrition is one of the most common complications in patients on maintenance HD and is associated with adverse clinical outcomes in these patients $[10,12]$. Many factors can affect the nutritional and metabolic status of these patients, including protein energy wasting, catabolic effects of renal replacement therapy, metabolic and hormonal disorders, and comorbidities. Several tools have been designed to evaluate nutritional status. The Subjective Global Assessment (SGA) and Malnutrition-Inflammation Score (MIS) are indicators of malnutrition for patients on maintenance HD [14, 15]. A previous study demonstrated that using modified quantitative SGA, malnutrition was found to be the most important factor associated with the amount of calcified depositions in the radial arteries among patients on maintenance HD [16]. However, the SGA and MIS require subjective assessment by the examiner, and compatibility between evaluations of different examiners is difficult to achieve. On the other hand, the GNRI is a simple tool that requires only serum albumin, height, and dry weight assessment. Several studies have reported that GNRI is the simplest and most accurate indicator of nutritional risk in patients on HD [11], and that it is a significant predictor of mortality in patients with CKD [17-20]. One previous study suggested that the optimal cutoff value of GNRI for mortality of HD patients was 90, based on the highest positive likelihood and risk ratios for overall survival [17]. Moreover, a recent observational study revealed that lower GNRI was significantly associated with severe vascular calcification in patients with CKD not on HD [12]. Indeed, the present study demonstrated that patients with rapid ACI progression had significantly lower GNRI than those with slow ACI progression (89.5 vs. 92.3). Furthermore, our results suggested that HD patients with GNRI $<90$ had an approximately 4-fold higher risk of rapid aortic calcification progression than those with GNRI $\geq 90$. In sum, our finding was consistent with previous reports, and suggested that poor nutritional status was closely associated with progression of arterial calcification in patients on maintenance HD.

Although the precise mechanism and relationship between progression of aortic calcification and malnutrition has not been fully elucidated, one possible explanation for the progression of aortic calcification is its association with malnutrition-inflammation-atherosclerosis (MIA) syndrome and/or fetuin-A. CKD-related inflammation causes malnutrition and progressive atherosclerosis, 
Table 1 Clinical characteristic of the $\triangle \mathrm{ACl}$-high and the $\triangle \mathrm{ACl}$-low groups

\begin{tabular}{|c|c|c|c|}
\hline & $\triangle \mathrm{ACl}$-high & $\triangle \mathrm{ACl}$-low & $P$-value \\
\hline Number & $56(30 \%)$ & $128(70 \%)$ & - \\
\hline Age $^{a}$ (year) & $68(58-77)$ & $65(58-74)$ & 0.149 \\
\hline Sex, male ${ }^{b}, n$ & 67 (53\%) & $41(27 \%)$ & 0.008 \\
\hline \multicolumn{4}{|l|}{ Cause of CKD } \\
\hline $\mathrm{DMN}^{\mathrm{b}}$ (presence), $\mathrm{n}$ & $31(55 \%)$ & $50(40 \%)$ & 0.04 \\
\hline Chronic glomerulonephritis $^{\mathrm{b}}$ (presence), $\mathrm{n}$ & $10(18 \%)$ & $27(21 \%)$ & 0.692 \\
\hline $\begin{array}{l}\text { Autosomal dominant polycystic kidney } \\
\text { disease }^{b} \text { (presence), } n\end{array}$ & $3(5.0 \%)$ & $5(4.0 \%)$ & 0.175 \\
\hline Others $^{b}$ (presence), $\mathrm{n}$ & $12(21 \%)$ & $46(36 \%)$ & 0.06 \\
\hline Modality of hemodialysis ${ }^{\mathrm{b}}$ & & & 1.00 \\
\hline$H D, n$ & $49(88 \%)$ & $111(87 \%)$ & - \\
\hline Online hemodiafiltration, $\mathrm{n}$ & $7(12 \%)$ & 17 (13\%) & - \\
\hline Systolic blood pressure $(\mathrm{mmHg})$ & $150(133-161)$ & $152(141-167)$ & 0.277 \\
\hline Diastolic blood pressure $(\mathrm{mmHg})$ & $78(69-91)$ & $78(72-86)$ & 0.776 \\
\hline $\mathrm{HTN}^{\mathrm{b}}$ (presence), $\mathrm{n}$ & $34(61 \%)$ & $97(76 \%)$ & 0.05 \\
\hline Current smoking ${ }^{\mathrm{b}}$ (presence) & $10(18 \%)$ & $16(14 \%)$ & 0.337 \\
\hline Previous history of $C V D^{\mathrm{b}}$ (presence), $\mathrm{n}$ & $14(25 \%)$ & $29(23 \%)$ & 0.710 \\
\hline $\mathrm{HD}^{\mathrm{a}}$ vintage (months) & $22.5(14.0-60.0)$ & $62.0(34.0-123)$ & $<0.001$ \\
\hline Serum albumin ${ }^{a}(g / d L)$ & $3.4(3.2-3.6)$ & $3.5(3.5-3.6)$ & 0.004 \\
\hline $\mathrm{CRP}^{\mathrm{a}}(\mathrm{mg} / \mathrm{dL})$ & $0.36(0.15-0.78)$ & $0.20(0.06-0.53)$ & 0.015 \\
\hline$\left.G N R\right|^{a}$ & $89.5(85.4-93.5)$ & $92.3(89.3-96.3)$ & 0.002 \\
\hline$G N R I^{b}<90, n$ & $31(55 \%)$ & $36(28 \%)$ & $<0.001$ \\
\hline $\mathrm{Kt} / \mathrm{N}^{\mathrm{a}}$ & $1.2(1.0-1.4)$ & $1.4(1.1-1.6)$ & $<0.001$ \\
\hline Serum phosphate ${ }^{\mathrm{a}}(\mathrm{mg} / \mathrm{dL})$ & $5.8(5.0-6.4)$ & $5.3(4.3-6.1)$ & 0.005 \\
\hline Corrected calcium ${ }^{\mathrm{a}}(\mathrm{mg} / \mathrm{dL})$ & $9.2(8.8-9.5)$ & $9.3(8.9-9.7)$ & 0.126 \\
\hline i-PTH $H^{a}(p g / m L)$ & $149(113-198)$ & $131(98-174)$ & 0.031 \\
\hline
\end{tabular}

Comparison values are median (interquartile range; IQR)

$A C l$ abdominal aortic calcification index, CKD chronic kidney disease, DMN diabetic nephropathy, HD hemodialysis, HTN hypertension, CVD cardiovascular disease, CRP C-reactive protein, GNRI Geriatric Nutrition Risk Index, i-PTH intact parathyroid hormone

${ }^{\text {a } M a n n-W h i t n e y ~ U-t e s t ~}$

${ }^{\mathrm{b}}$ Fisher's exact test

which is known as MIA syndrome [21]. Fetuin-A, a circulating calcium-regulatory glycoprotein, was reported to be closely associated with MIA syndrome [22]. Fetuin-A inhibits vascular calcification in patients with CKD by removing calciprotein particles (calcium phosphate-containing nano-aggregates) [23]. Both the activity and serum levels of fetuin-A are decreased in dialyzed patients [24]. Furthermore, previous reports have shown that fetuin-A levels were positively correlated with serum albumin, serum prealbumin, and SGA in dialyzed patients [25-27]. Our results regarding the relationship between malnutrition and rapid aortic calcification progression may support these findings. However, few studies have provided sufficient evidence of the effectiveness of nutritional management to increase fetuin-A levels and decrease progression of vascular calcification. Further prospective studies will be needed to determine that nutrition treatment for HD patients can prevent progression of vascular calcification.

Several studies have reported that patients on maintenance HD experience more rapid progression of vascular calcification than do healthy individuals [28]. Our results suggested that classical and non-classical risk factors had a significant impact on aortic calcification progression. These findings were consistent with previous studies which demonstrated that male sex $[29,30]$, hyperphosphatemia [6-8] accelerate the process of vascular calcification and atherosclerosis among patients with CKD and/or who are on maintenance HD. On the other hand, the relationship between HD vintage and progression of vascular calcification has been controversial. Previous studies have revealed that dialysis vintage was positively correlated with vascular calcification progression [29, 31]. However, another study demonstrated the converse relationship [32]. 


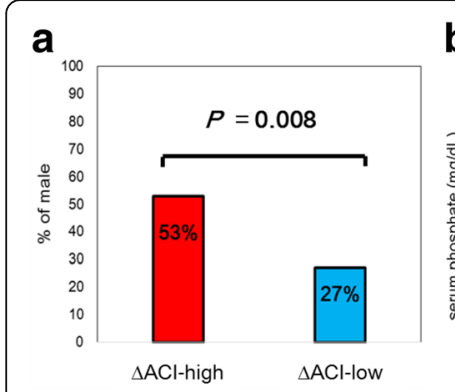

b

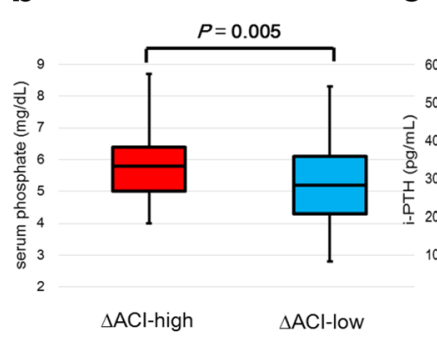

C

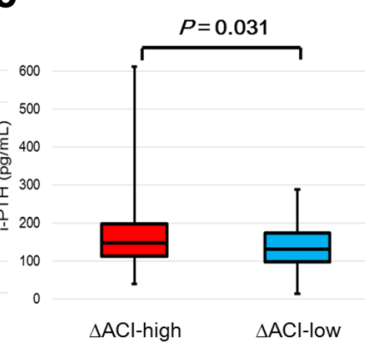

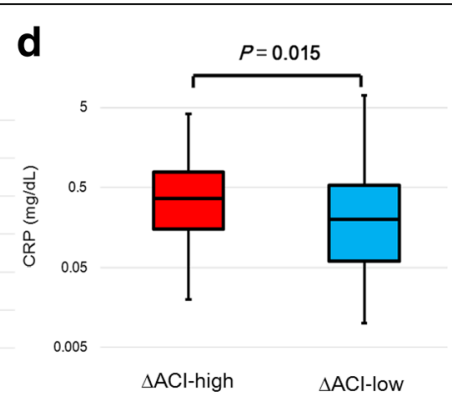
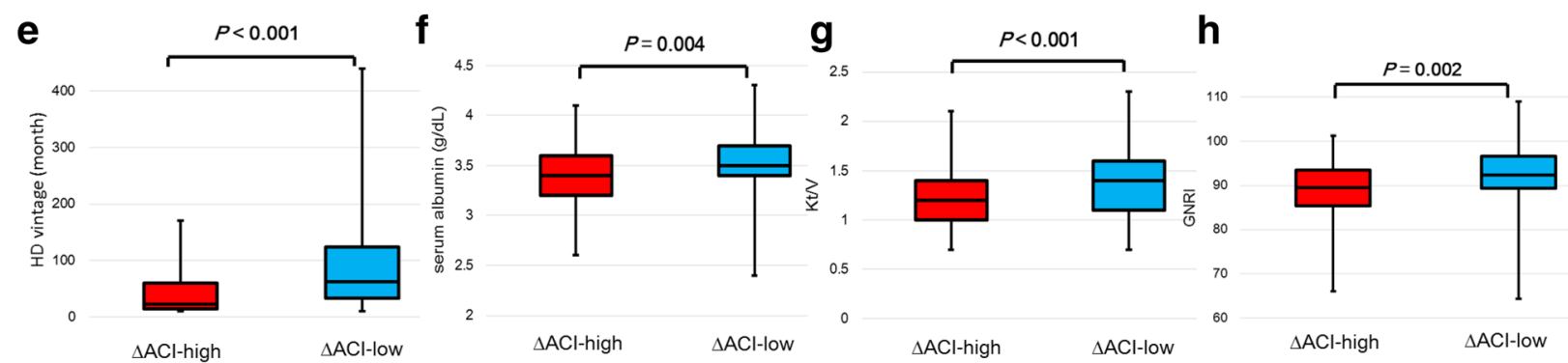

Fig. 3 Comparison of clinical characteristics between the $\triangle \mathrm{ACl}$-high and $\triangle \mathrm{ACl}$-low groups. In the $\triangle \mathrm{ACl}$-high group $(\Delta \mathrm{ACl} \geq 5.8 \%)$, the proportion of males was significantly higher compared with the $\Delta \mathrm{ACl}$-low group $(\Delta \mathrm{ACl}<5.8 \%)(\mathbf{a})$. Among patients in the $\Delta \mathrm{ACl}$-high group, serum phosphate $(\mathbf{b})$, intact parathyroid hormone (i-PTH) (c), and CRP (d) were significantly higher compared with patients in the $\triangle$ ACl-low group. Hemodialysis (HD) vintage (e), serum albumin (f), KtN (g), and Geriatric Nutritional Risk Index (GNRI) (h) were significantly shorter or lower in the $\Delta$ ACl-high group than in the $\triangle \mathrm{ACl}$-low group

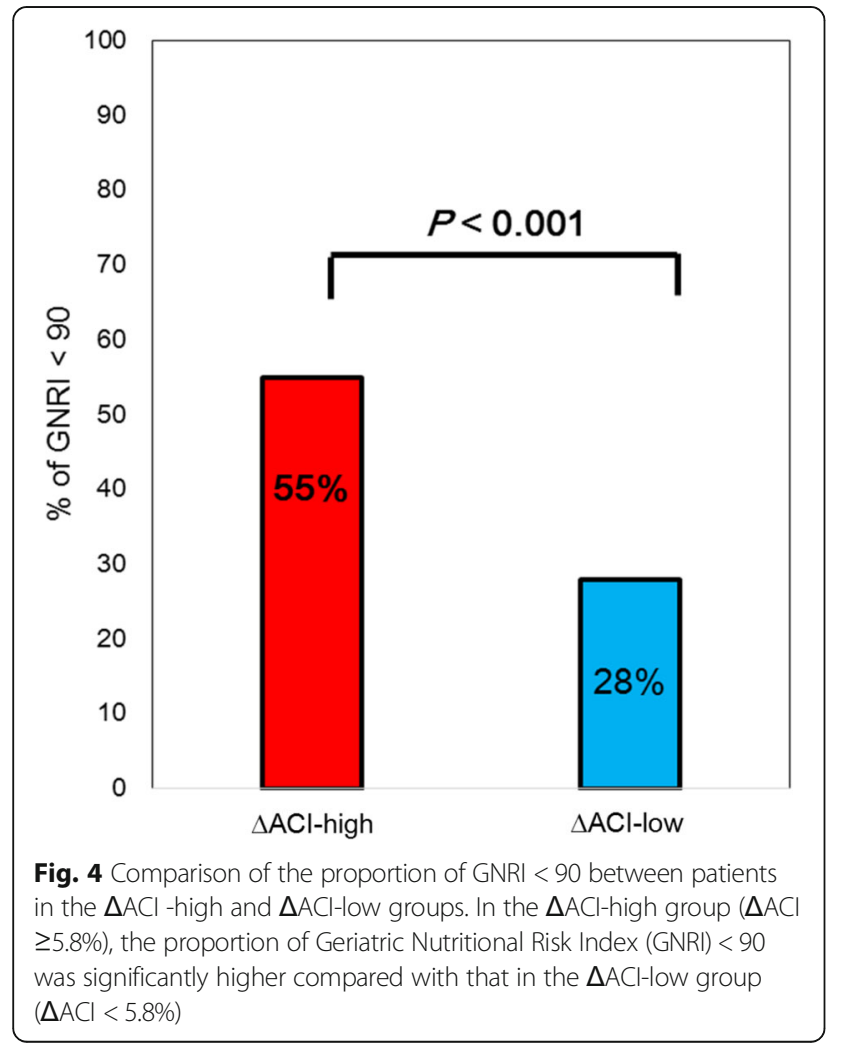

In the present study, patients with rapid progression of ACI had significantly shorter HD vintage than those with slow progression (22.5 vs 62.0 months). A previous study demonstrated that the initiation of hemodialysis triggered apoptosis of vascular smooth muscle cell, which induced rapid and extreme vascular calcification [33]. This finding may imply that rapid progression of vascular calcification occurred in the early transition period following initiation of HD. Our previous study revealed that even after renal transplantation, many patients who experienced maintenance HD showed significant progression of aortic

Table $\mathbf{2}$ Independent risk factors for high $\triangle \mathrm{ACl}$ by multivariate logistic regression analysis

\begin{tabular}{lllll}
\hline Variable & Risk factor & $P$-value & Odds ratio & $95 \% \mathrm{Cl}$ \\
\hline GNRI & $<90$ & 0.001 & 4.17 & $1.79-9.71$ \\
Sex & Male & 0.014 & 3.29 & $1.27-8.53$ \\
Current smoking & Positive & 0.197 & 2.08 & $0.68-6.35$ \\
Serum phosphate (mg/dL) & Continuous & 0.004 & 1.71 & $1.18-2.47$ \\
DMN & Positive & 0.645 & 1.26 & $0.53-2.78$ \\
corrected calcium (mg/dL) & Continuous & 0.694 & 1.18 & $0.53-2.63$ \\
i-PTH (ng/mL) & Continuous & 0.055 & 1.01 & $1.00-1.01$ \\
Age (year) & Continuous & 0.472 & 1.01 & $0.98-1.05$ \\
HTN & Positive & 0.078 & 0.47 & $0.20-1.09$ \\
HD vintage (month) & Continuous & 0.007 & 0.99 & $0.98-0.99$
\end{tabular}

GNRI Geriatric Nutrition Risk Index, DMN Diabetic nephropathy, i-PTH intact parathyroid hormone, HTN Hypertension, HD hemodialysis 


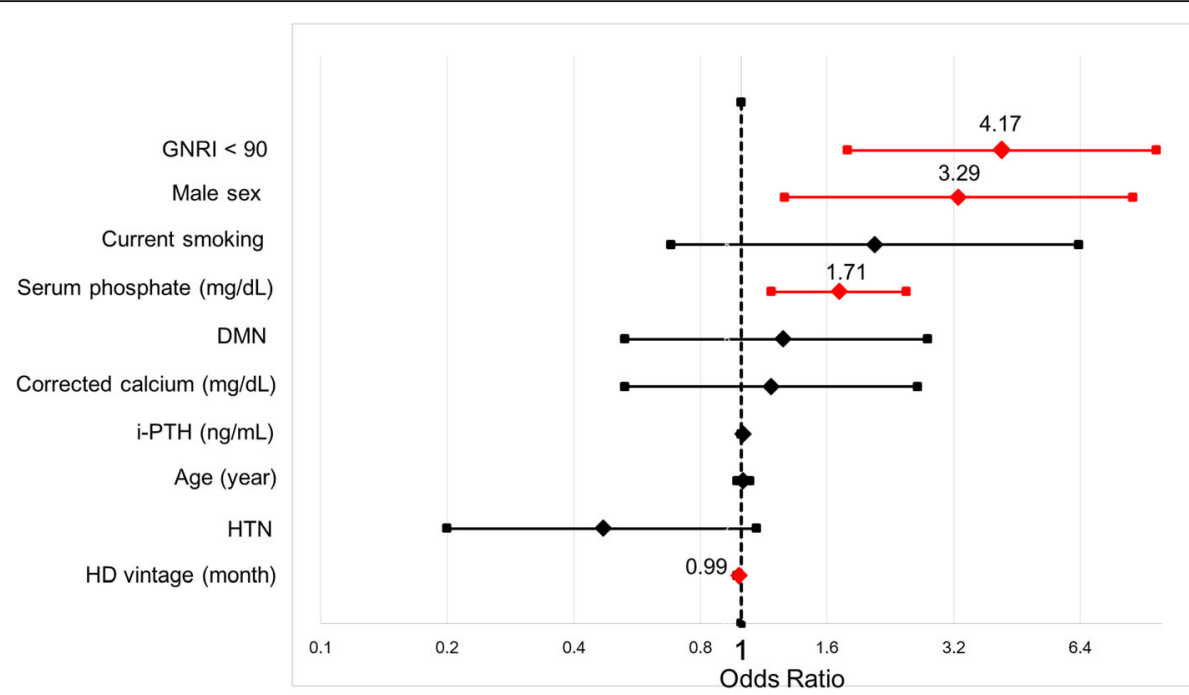

Fig. 5 Factors associated with $\mathrm{ACl}$ progression. Independent risk factors for high $\triangle \mathrm{ACl}$ were evaluated using multivariate logistic regression analysis. Geriatric Nutritional Risk Index (GNRI) < 90, male sex, serum phosphate, and hemodialysis (HD) vintage were selected as independent risk factors for $\mathrm{ACl}$ progression

calcification for 10 years [34]. This finding may imply that the progression of vascular calcification was significantly enhanced by the presence of CKD. Moreover, progression of vascular calcification is closely related with poor all-cause and cardiovascular mortality-free survival rates $[35,36]$. Because there is a complex web of interactions between the progression of vascular calcification and several risk factors, the mechanism of vascular calcification remains unclear.

Several limitations of this study should be described. First, this study was conducted retrospectively at a single center. In addition, its small sample size and selection biases prevent us from obtaining definitive conclusions. Second, our semi-quantitative measurement of aortic calcification did not enable us to evaluate its change in thickness. Third, we could not address the impact of medications such as phosphate binders, cinacalcet, and antihypertensive drugs, nor of dietary habits and normalized protein catabolic rates, which represent protein intake. Finally, because the GNRI requires only serum albumin, body weight, and height, it does not reflect total body composition, which consists of muscle mass, fat, and total body water. Despite these limitations, using a simple and accurate nutritional assessment tool, we were able to demonstrate an independent association between malnutrition and progression of aortic calcification in patients on maintenance HD. In our next study, we plan to address the relationship between malnutrition, fetuin-A, and aortic calcification.

\section{Conclusions}

A potential role between malnutrition and aortic calcification may exist in patients on maintenance HD. Our findings may encourage clinicians to pay greater attention to nutrition management to prevent the progression of arterial calcification.

\section{Abbreviations}

ACl: Abdominal aortic calcification index; CKD: Chronic kidney disease; CRP: C-reactive protein; CT: Computed tomography; CVD: Cardiovascular disease; DMN: Diabetic nephropathy; GNRI: Geriatric nutritional risk index; HD: Hemodialysis; HTN: Hypertension; i-PTH: Intact parathyroid hormone; MIA: Malnutrition-inflammation-atherosclerosis; MIS: Malnutritioninflammation score; SGA: Subjective global assessment

\section{Acknowledgments}

We thank Shizuka Hosoya and Kaori Yamagami for their invaluable help with the data collection.

\section{Funding}

This work was supported by the Japan Society for the Promotion of Science (No. 15 K15579, 17 K11119).

\section{Availability of data and materials}

The datasets are not publicly available but are available from the first author on reasonable request.

\section{Authors' contributions}

TO performed the statistical analysis and drafted the manuscript. $\mathrm{SH}^{2}$ assisted in drafting the manuscript. SH ${ }^{1,2} \mathrm{YT}, \mathrm{T}$, and FS supported the clinical follow-up. HK, HH, YK, KK, and MM helped in collecting data for the manuscript. ST and CO supervised this study. $\mathrm{SH}^{2}$ was responsible for the interpretation of data and critical revision of the manuscript. All authors read and approved the final manuscript.

Ethics approval and consent to participate

The study was conducted in accordance with the ethical standards of the Declaration of Helsinki and was approved by the Ethics Committee of Hirosaki University Graduate School of Medicine (authorization number 2016-225). For this type of retrospective study, formal patient consent is not required.

Consent for publication Not applicable. 


\section{Competing interests}

The authors declare that they have no competing interests.

\section{Publisher's Note}

Springer Nature remains neutral with regard to jurisdictional claims in published maps and institutional affiliations.

\section{Author details}

'Department of Urology, Oyokyo Kidney Research Institute Aomori Hospital, 101-1 Okabe, Aomori 038-0003, Japan. ²Department of Urology, Hirosaki University Graduate School of Medicine, 5 Zaifu-chou, Hirosaki 036-8562, Japan. ${ }^{3}$ Department of Urology, Oyokyo Kidney Research Institute, Hirosaki, Japan. ${ }^{4}$ Department of Advanced Transplant and Regenerative Medicine, Hirosaki University Graduate School of Medicine, Hirosaki, Japan.

Received: 8 July 2017 Accepted: 14 March 2018

Published online: 20 March 2018

\section{References}

1. Blacher J, Guerin AP, Pannier B, Marchais SJ, London GM. Arterial calcifications, arterial stiffness, and cardiovascular risk in end-stage renal disease. Hypertension. 2001;38:938-42

2. Garland JS, Holden RM, Groome PA, Lam M, Nolan RL, Morton AR, et al. Prevalence and associations of coronary artery calcification in patients with stage3 to CKD without cardiovascular disease. Am J Kidney Dis. 2008:52:849-58

3. Witteman JC, Kok FJ, van Saase JL, Valkenburg HA. Aortic calcification as a predictor of cardiovascular mortality. Lancet. 1986;2:1120-2.

4. Davies MR, Hruska KA. Pathophysiological mechanisim of vascular calcification in end-stage renal disease. Kidney Int. 2001;60:472-9.

5. Chen NC, Hsu CY, Chen CL. The strategy to prevent and regress the vascular calcification in dialysis patients. Biomed Res Int. 2017;2017:9035193. https://doi.org/10.1155/2017/9035193.

6. Goldsmith D, Ritz E, Covic A. Vascular calcification: a stiff challenge for the nephrologist: does preventing bone disease cause arterial disease? Kidney Int. 2004:66:1315-33.

7. Block GA, Hulbert-Shearon TE, Levin NW, Port FK. Association of serum phosphorus and calcium $x$ phosphate product with mortality risk in chronic hemodialysis patients: a national study. Am J Kidney Dis. 1998;31:607-17.

8. Yamamoto D, Suzuki S, Ishii H, Hirayama K, Harada K, Aoki T, et al. Predictors of abdominal aortic calcification progression in patients with chronic kidney disease without hemodialysis. Atherosclerosis. 2016;253:15-21.

9. Toussaint ND, Lau KK, Strauss BJ, Polkinghorne KR, Kerr PG. Effect of alendronate on vascular calcification in CKD stages 3 and 4: a pilot randomized controlled trial. Am J Kidney Dis. 2010:56:57-68.

10. Ishii H, Takahashi H, Ito Y, Aoyama T, Kamoi D, Sakakibara T, et al. The Association of Ankle Brachial Index, protein-energy wasting, and inflammation status with cardiovascular mortality in patients on chronic hemodialysis. Nutrients. 2017:9(4):416. https://doi.org/10.3390/nu9040416.

11. Yamada K, Furuya R, Takita T, Maruyama Y, Yamaguchi Y, Ohkawa S, et al. Simplified nutritional screening tools for patients on maintenance hemodialysis. Am J Clin Nutr. 2008:87:106-13.

12. Harada K, Suzuki S, Ishii H, Hirayama K, Aoki T, Shibata Y, et al. Nutrition status predicts severity of vascular calcification in non-dialyzed chronic kidney disease. Circ J. 2017;81:316-21.

13. Tsushima M, Terayama Y, Momose A, Funyu T, Ohyama C, Hada R. Carotid intima media thickness and aortic calcification index closely relate to cerebro- and cardiovascular disorders in hemodialysis patients. Int J Urol. 2008;15:48-51.

14. Detsky AS, McLaughlin JR, Baker JP, Johnston N, Whittaker S, Mendelson RA, et al. What is subjective global assessment of nutritional status? J Parenter Enter Nutr. 1987;11:8-13.

15. Kalantar ZK, Kopple JD, Block G, Humphreys MH. A malnutritioninflammation score is correlated with morbidity and mortality in maintenance hemodialysis patients. Am J Kidney Dis. 2001:38:1251-63.

16. Zhang K, Cheng G, Cai X, Chen J, Jiang Y, Wang T, et al. Malnutrition, a new inducer for arterial calcification in hemodialysis patients? J Transl Med. 2013; 11:66. https://doi.org/10.1186/1479-5876-11-66.

17. Kobayashi I, Ishimura E, Kato Y, Okuno S, Yamamoto T, Yamakawa T, et al. Geriatric nutritional risk index, a simplified nutritional screening index, is a significant predictor of mortality in chronic dialysis patients. Nephrol Dial Transplant. 2010:25:3361-5.

18. Kiuchi A, Ohashi Y, Tai R, Aoki T, Mizuiri S, Ogura T, et al. Association between low dietary protein intake and geriatric nutrition risk index in patients with chronic kidney disease: a retrospective single-center cohort study. Nutrients. 2016;8:662. https://doi.org/10.3390/nu8100662.

19. Kang SH, Cho KH, Park JW, Yoon KW, Do JY. Geriatric nutritional risk index as a prognostic factor in peritoneal dialysis patients. Perit Dial Int. 2013:33:405-10

20. Panichi V, Cupisti A, Rosati A, Di Giorgio A, Scatena A, Menconi O, et al. Geriatric nutritional risk index is a strong predictor of mortality in hemodialysis patients: data from the Riscavid cohort. J Nephrol. 2014;27: 193-201.

21. Stenvinkel P. Inflammatory and atherosclerotic interactions in the depleted uremic patient. Blood Purif. 2001;19:53-61.

22. Ketteler M, Bongartz $\mathrm{P}$, Westenfeld R, Wildberger JE, Mahnken AH, Böhm R, et al. Association of low fetuin-a (AHSG) concentrations in serum with cardiovascular mortality in patients on dialysis: a cross-sectional study. Lancet. 2003;361:827-33.

23. Suliman ME, García-López E, Anderstam B, Lindholm B, Stenvinkel P. Vascular calcification inhibitors in relation to cardiovascular disease with special emphasis on fetuin-a in chronic kidney disease. Adv Clin Chem. 2008;46:217-62

24. Herrmann M, Schafer C, Heiss A, Gräber S, Kinkeldey A, Büscher A, et al. Clearance of fetuin-A-containing calciprotein particles is mediated by scavenger receptor-a. Circ Res. 2012;111:575-84.

25. Oikawa O, Higuchi T, Yamazaki T, Yamamoto C, Fukuda N, Matsumoto K. Evaluation of serum fetuin-a relationships with biochemical parameters in patients on hemodialysis. Clin Exp Nephrol. 2007;11:304-8.

26. Coen G, Manni M, Agnoli A, Balducci A, Dessi M, De Angelis S, et al. Cardiac calcifications: Fetuin-a and other risk factors in hemodialysis patients. ASAIO J. 2006;52:150-6.

27. Wang AY, Woo J, Lam CW, Wang M, Chan IH, Gao P, et al. Associations of serum fetuin-a with malnutrition, inflammation, atherosclerosis and valvular calcification syndrome and outcome in peritoneal dialysis patients. Nephrol Dial Transplant. 2005;20:1676-85

28. Goodman W, Goldin J, Kuizon BD, Yoon C, Gales B, Sider D, et al. Coronaryartery calcification in young adults with end-stage renal disease who are undergoing dialysis. N Engl J Med. 2000;342:1478-83.

29. Taniwaki H, Ishimura E, Tabata T, Tsujimoto Y, Shioi A, Shoji T, et al. Aortic calcification in haemodialysis patients with diabetes mellitus. Nephrol Dial Transplant. 2005:20:2472-8.

30. Mhairi S, Maarten T, Peter B, McIntyre CW. Progressive vascular calcification over 2 years is associated with arterial stiffening and increased mortality in patients with stages 4 and 5 chronic kidney disease. Clin J Am Soc Nephrol. 2007;2:1241-8

31. Kim H, Song S, Kim T, Kim YO. Risk factors for progression of aortic arch calcification in patients on maintenance hemodialysis and peritoneal dialysis. Hemodial Int. 2011;15:460-7.

32. Yamada K, Fujimoto $S$, Nishiura R, Komatsu H, Tatsumoto M, Sato $Y$, et al. Risk factors of the progression of abdominal aortic calcification in patients on chronic haemodialysis. Nephrol Dial Transplant. 2007;22(7):2032.

33. Shroff RC, McNair R, Figg N, Skepper JN, Schurgers L, Gupta A, et al. Dialysis accelerates medial vascular calcification in part by triggering smooth muscle cell apoptosis. Circulation. 2008;118:1748-57.

34. Imanishi K, Hatakeyama S, Yamamoto H, Okamoto A, Imai A, Yoneyama T, et al. Post-transplant renal function and cardiovascular events are closely associated with the aortic calcification index in renal transplant recipients. Transplant Proc. 2014:46:484-8.

35. Noordzij M, Cranenburg EM, Engelsman LF, Hermans MM, Boeschoten EW, Brandenburg VM, et al. Progression of aortic calcification is associated with disorders of mineral metabolism and mortality in chronic dialysis patients. Nephrol Dial Transplant. 2011;26:1662-9.

36. Lee MJ, Shin DH, Kim SJ, Oh HJ, Yoo DE, Ko Kl, et al. Progression of aortic arch calcification over 1 year is an independent predictor of mortality in incident peritoneal dialysis patients. PLoS One. 2012;7:e48793. 